\title{
Sensing Characteristics of Side-Hole Fiber-Based Long-Period Grating
}

\author{
Yi Xin, ${ }^{1}$ Xinyong Dong, ${ }^{1}$ Junwei Yuan, ${ }^{1}$ Yi Li, ${ }^{1}$ Shangzhong Jin, ${ }^{1}$ and Sulei Zhang ${ }^{2}$ \\ ${ }^{1}$ Institute of Optoelectronic Technology, China Jiliang University, Hangzhou 310018, China \\ ${ }^{2}$ Civil Engineering School, Zhengzhou University, Zhengzhou 450001, China \\ Correspondence should be addressed to Xinyong Dong; dong_X_y@hotmail.com
}

Received 22 May 2013; Accepted 20 July 2013

Academic Editor: Sulaiman Wadi Harun

Copyright (c) 2013 Yi Xin et al. This is an open access article distributed under the Creative Commons Attribution License, which permits unrestricted use, distribution, and reproduction in any medium, provided the original work is properly cited.

Long-period gratings (LPGs) have been fabricated in a side-hole fiber (SHF) by using a pulsed $\mathrm{CO}_{2}$ laser. Sensing characteristics of this SHF-LPG to temperature surrounding refractive index and bend have been investigated. Experimental results show that resonant wavelength of the SHF-LPG has a blue shift with temperature with sensitivity of $-0.11 \mathrm{~nm} /{ }^{\circ} \mathrm{C}$, a blue shift with increasing sensitivity with surrounding refractive index ranging from 1.335 to 1.44 (the maximum sensitivity is achieved when the surrounding refractive index reaches the effective index of the fiber cladding), and a red shift with bend-direction-dependent sensitivity up to $9.36 \mathrm{~nm} / \mathrm{m}^{-1}$.

\section{Introduction}

Side-hole fiber (SHF) is a highly-birefringent fiber with two air holes running in parallel to the core in the cladding. It was first proposed by Xie et al. as a possible sensor for hydrostatic or acoustic pressure measurement [1]. The measurement is based on the anisotropic elastic stress and photoelastic effect introduced by pressure, and the pressure can be applied from inside of the air holes or from outside of the fiber $[1,2]$. SHF may also have high temperature sensitivity with $\mathrm{dB} / \mathrm{dT}$ up to $10^{-7} /{ }^{\circ} \mathrm{C}$ by controlling the amount of germanium doped in the core region and optimizing the distance between the core and side holes [3]. It is at least one order higher than that of standard polarization maintaining fibers $(\mathrm{dB} / \mathrm{dT}$ $\sim 10^{-8} /{ }^{\circ} \mathrm{C}$ ). In recent years, special sensing applications have been investigated by filling air holes of SHF with liquid, gas, and even metal [4-8].

Fiber gratings, with a periodic refractive index (RI) distribution in the axial direction of the fiber, are widely applied in fiber sensing and optical communication due to their many advantages such as simple structure, high sensitivity, wellcontrolled wavelength-selective capability, and capability of remote interrogation of multiple sensors [9-14]. SHF-based fiber grating devices have attracted lots of research interest
[11-13]. Simultaneous measurement of strain and temperature has been reported by using a fiber Bragg grating (FBG) recorded on the fusion splicing joint between an SHF and a single-mode fiber (SMF) [11]. Simultaneously measurement of pressure and temperature has also been realized by using an FBG imprinted on the SHF with an elliptical core [12]. In our previous study, a long-period grating (LPG) has been introduced mechanically in an SHF and been applied to temperature and RI measurements [13]. However, it is not convenient in practical applications because the mechanical part cannot be removed. Otherwise the LPG will disappear.

In this paper, LPGs are fabricated by using pulsed $\mathrm{CO}_{2}$ laser in the SHF with a cylindrical core, and their sensing characteristics to temperature surrounding refractive index and bend are investigated. The sensor fabrication and principle are presented in Section 2. The experiment results and discussions are shown in Section 3, and a conclusion is given in Section 4.

\section{Sensor Fabrication and Principle}

The schematic diagram of the $\mathrm{CO}_{2}$ laser system for LPG fabrication is shown in Figure 1. It consists of a broad band source (BBS) with a wavelength resolution of $0.1 \mathrm{~nm}$, a $10 \mathrm{~cm}$ 


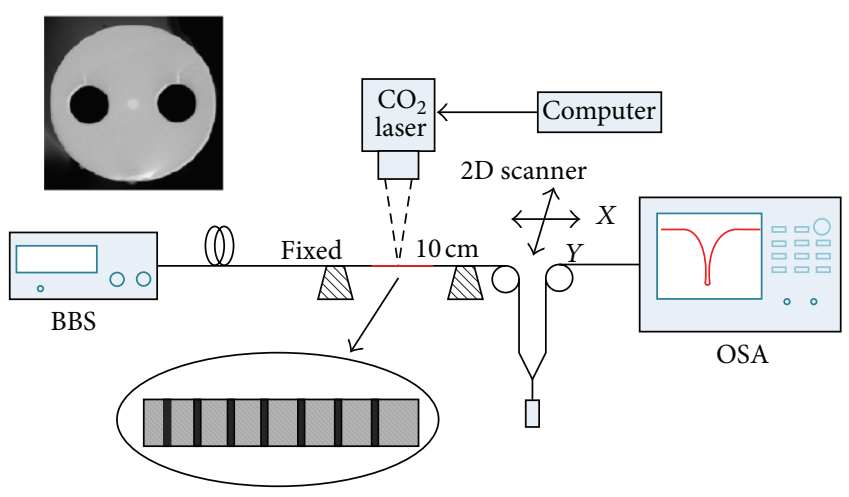

FIGURE 1: Schematic diagram of the $\mathrm{CO}_{2}$ laser system for LPG fabrication.

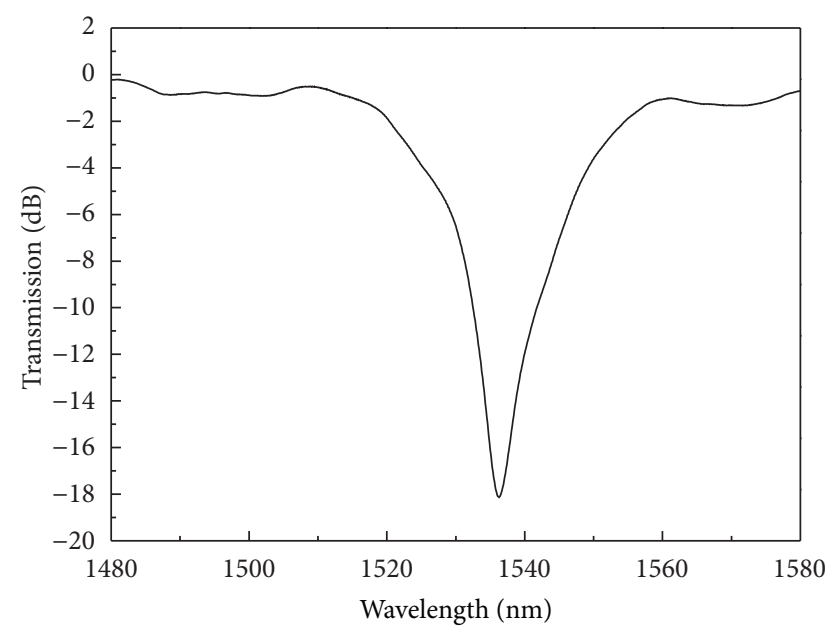

Figure 2: Transmission spectrum of the SHF-LPG fabricated using $\mathrm{CO}_{2}$ laser.

long SHF, and an optical spectrum analyzer (OSA) with a $3 \mathrm{~dB}$ bandwidth of $62 \mathrm{~nm}$. The SHF used in the experiment, with a scanning electron micrograph (SEM) of the crosssection shown in the insert of Figure 1, has core and cladding diameters of $8 \mu \mathrm{m}$ and $125 \mu \mathrm{m}$, respectively. The two air holes with the same diameter of $28 \mu \mathrm{m}$ are located symmetrically in the cladding aside the core. The distance between the centers of the air holes and the core is $32 \mu \mathrm{m}$. The difference in RI between the core and cladding is 0.0056 [15]. The $\mathrm{CO}_{2}$ laser system, with a maximum output power of $10 \mathrm{~W}$ and Q-switch time of $105 \mu \mathrm{s}$, operates at a typical frequency of $5 \mathrm{kHz}$, which is probably the best frequency for glass to absorb the energy of the $\mathrm{CO}_{2}$ laser. The fiber is scanned at both $x$ - and $y$-axes by means of a two-dimensional optical scanner attached to the laser head. Figure 2 shows the transmission spectrum of the so-formed LPG with period of $520 \mu \mathrm{m}$ and length of $21.32 \mathrm{~mm}$ by 12 scanning cycles. The resonant wavelength of this LPG is $1536.3 \mathrm{~nm}$ with a notch depth of $18 \mathrm{~dB}$.

The LPG couples light power between the fundamental guided mode and forward-propagating cladding modes inside the fiber. As a result, several resonant modes may appear in the corresponding transmission spectrum.
The resonant wavelength of $m$ th order mode is defined by the phase-matching condition [16]

$$
\lambda_{m}=\left(n_{\text {core }}-n_{\text {clad }}^{m}\right) \Lambda
$$

where $\Lambda$ is the grating period; $n_{\text {core }}$ and $n_{\text {clad }}^{m}$ are the effective indices of the fundamental guided mode and the $m$ th order cladding modes, respectively. The resonant wavelength of LPG can be expressed as [16]

$$
\lambda_{\text {max }}=\lambda_{\text {res }}\left[1+\frac{\delta n_{\text {core }}\left(\mathrm{d} \lambda_{\text {res }} / \mathrm{d} \Lambda\right)}{\left(n_{\text {core }}-n_{\text {cl }}^{m}\right)^{2}}\right],
$$

where $\delta n_{\text {core }}$ is the average effective RI variation of the fundamental guided mode. The resonant wavelength and amplitude of LPG are sensitive to the change of average effective RI for every period of fiber core and fiber cladding, as coupling mode is very sensitive to perturbation of guided mode and cladding mode. Therefore, the change of external parameters, such as temperature, strain, bend, and RI, can be determined by measuring resonant wavelength shift of the SHF-LPG.

\section{Experimental Results and Discussions}

3.1. Temperature Sensing Characteristics of SHF-LPG. Temperature response of the SHF-LPG was tested by placing it into an oven and changing the temperature. The SHF-LPG was pasted on a capillary to prevent from the influence of strain and bend. During the measurement, the spectrum was recorded with a temperature interval of $10^{\circ} \mathrm{C}$ between $20^{\circ} \mathrm{C}$ and $80^{\circ} \mathrm{C}$. Before each record, the temperature was maintained for $10 \mathrm{~min}$ to ensure its accuracy.

Figure 3(a) shows measured transmission spectra of the SHF-LPG under different temperatures. As temperature increases from $20^{\circ} \mathrm{C}$ to $80^{\circ} \mathrm{C}$, the resonant wavelength has a blue shift from $1532.68 \mathrm{~nm}$ to $1526.17 \mathrm{~nm}$. Figure 3(b) shows the resonant wavelength response to temperature. The achieved temperature sensitivity is $-0.11 \mathrm{~nm} /{ }^{\circ} \mathrm{C}$ with $R$ squared value of 0.984 . The sensitivity is about 2 times higher than that of SMF-LPG written by $\mathrm{CO}_{2}$ laser [17], 1.35 times higher than that of mechanically-introduced SHF-LPG [13], and ten times higher than that of normal FBGs [3]. The resonant wavelength shifted to shorter wavelength because RI difference, $\Delta n\left(\Delta n=n_{\text {core }}-n_{\text {clad }}^{m}\right)$, decreases with temperature. The thermal optic coefficient of the cladding is larger than that of the fiber core. The amplitude varies slightly with temperature that may be caused by the pneumatic impact on the oven. However, it does not affect the measurement accuracy because the measurement is based on wavelength, not intensity.

3.2. Refractive Index Characteristics of SHF-LPG. The RI sensitivity was measured using sugar solution with different concentrations. The corresponding calibrated RI accuracy for each liquid is $\pm 0.002 \mathrm{RIU}$. The SHF-LPG was put in groove of Plexiglas, applying a small prestressing force to straighten it. The resonant wavelength was recorded when the SHF-LPG was coated by liquid with a different surrounding RI. All the experiments were carried out in a controlled 


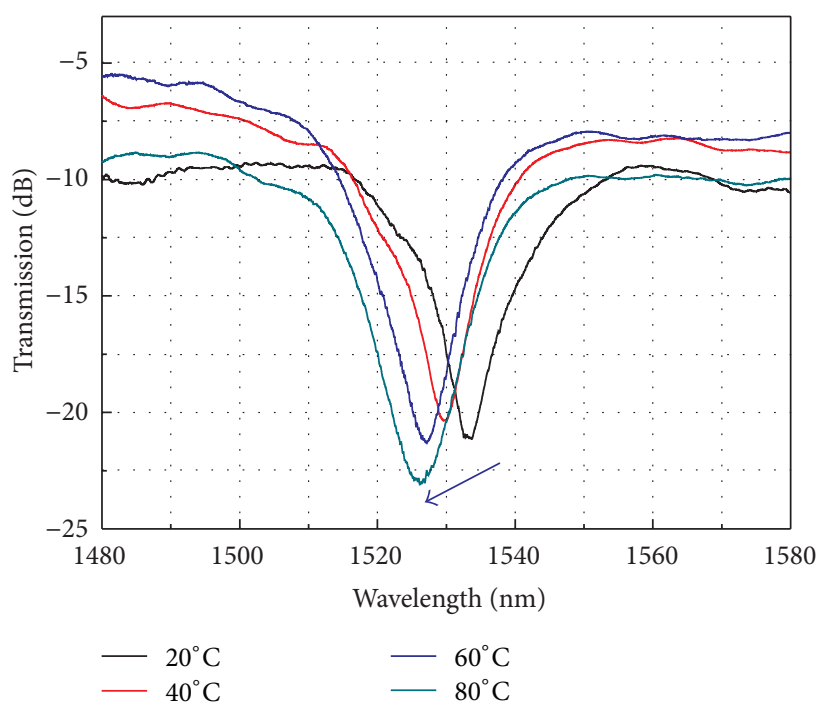

(a)

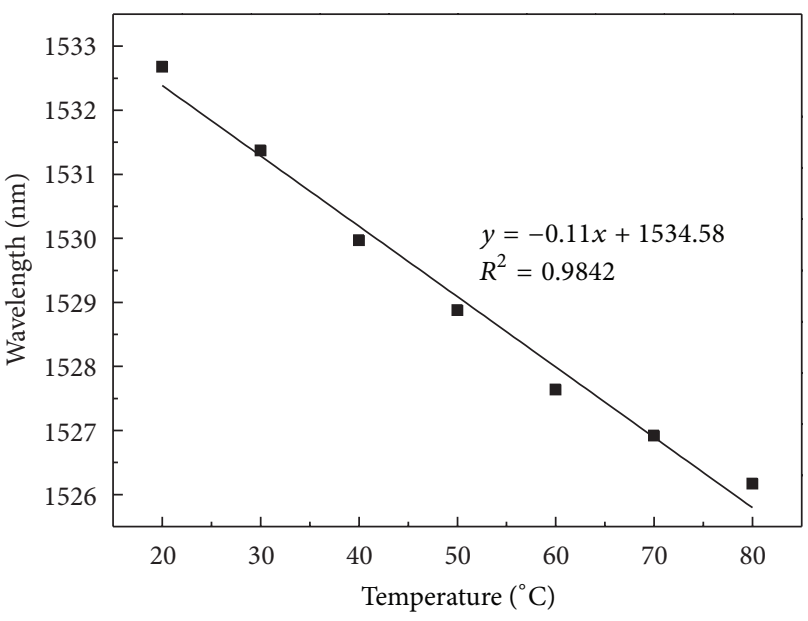

(b)

Figure 3: (a) Transmission spectra of the SHF-LPG under different temperatures. (b) Resonant wavelength versus temperature.

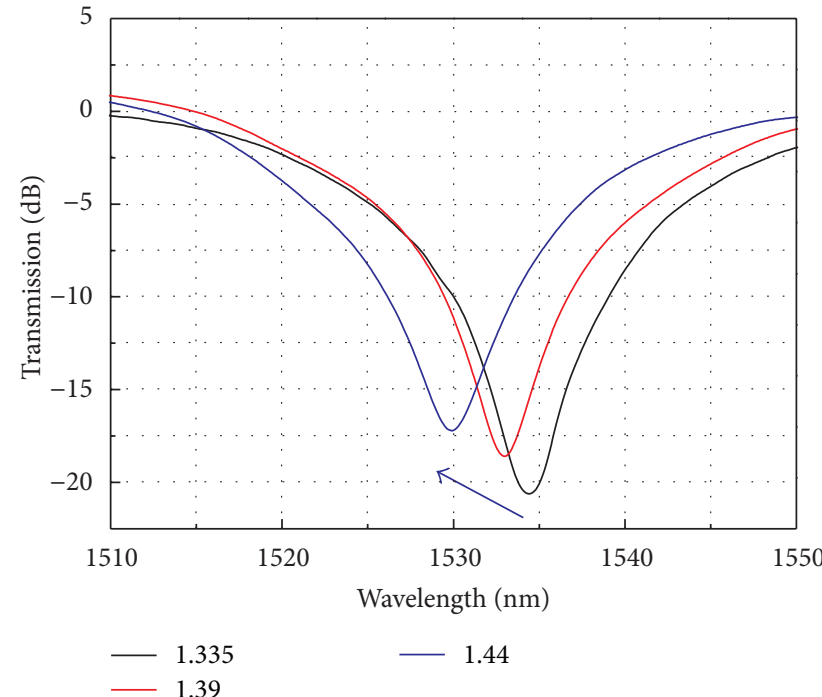

(a)

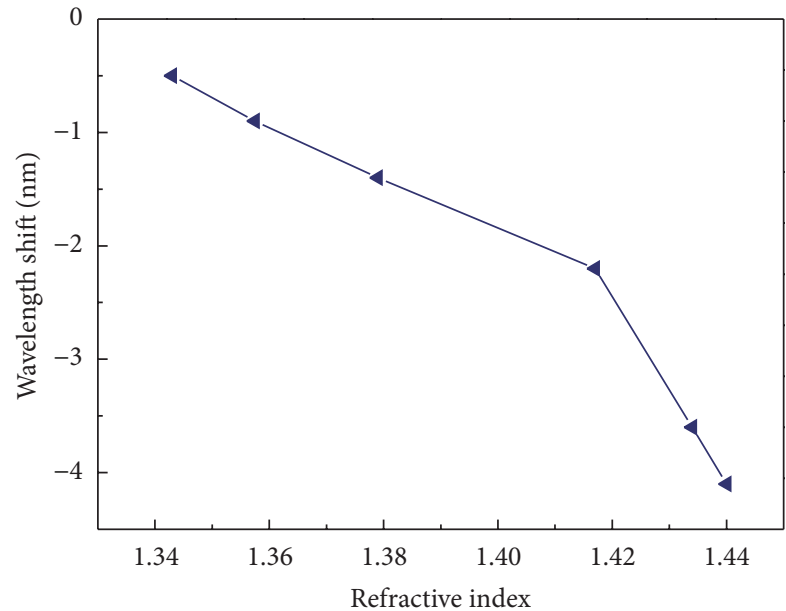

(b)

FIgURE 4: Transmission spectra of the SHF-LPG under different RIs. (b) Resonant wavelength versus RI.

environment, where the effect of temperature variation can be ignored.

Figure 4(a) shows measured transmission spectra of the SHF-LPG under different surrounding RIs of $1.335,1.39$, and 1.44. The resonant wavelength exhibits blue shift, and the amplitude reduces with surrounding RI. The resonant wavelength had a shift of $4.5 \mathrm{~nm}$, from $1534.4 \mathrm{~nm}$ to $1529.9 \mathrm{~nm}$, when surrounding RI was changed from 1.335 to 1.44 . Figure 4(b) shows the resonant wavelength shift against surrounding RI. The resonant wavelength exhibits a blue shift of $1.4 \mathrm{~nm}$ for the RI changing from 1.335 to 1.39 and a blue shift of $3.1 \mathrm{~nm}$ for the RI changing from 1.39 to 1.44 . The maximum sensitivity is achieved when the surrounding RI reaches the effective index of the fiber cladding. The effective
RI of cladding modes increases with surrounding RI; thus the RI difference, $\Delta n$, decreases. That makes the resonant wavelength of SHF-LPG blue shifts as (1) indicates.

In this case, the RI-induced resonant wavelength shift is relatively small if it is compared with those of the SMFLPG fabricated with the same method [18] and of the mechanically-introduced SHF-LPG [13]. The relatively lower RI sensitivity of SHF-LPG than SMF-LPG may be related to the relatively lower cladding effective RI of the SHF than SMF caused by the existence of the two air holes. According to [19], higher effective RI of fiber cladding may produce higher sensitivity of LPG to surrounding RI. As for mechanicallyintroduced SHF-LPG, the RI measurement sensitivity is higher than ours because it was carried out by infiltrating 


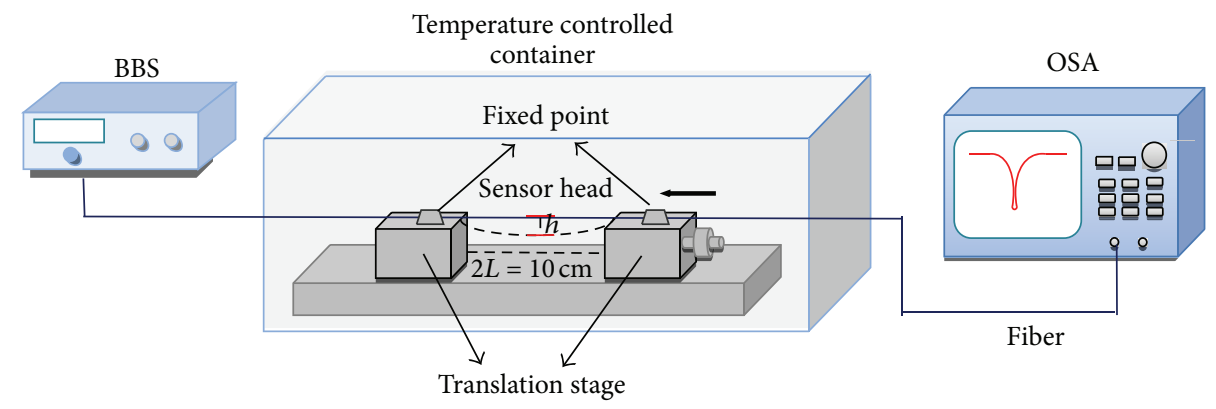

FIGURE 5: Schematic diagram of bend measurement.

the tested liquid directly into air holes of the fiber; thus its modulation to effective RI of fiber cladding is better. However, the infiltrating method is not convenient in practical operation because the SHF cannot be fusion-spliced to other fibers.

3.3. Bend Characteristics of SHF-LPG. Figure 5 shows the schematic diagram of the experimental setup for bend measurement using the SHF-LPG. Bend test was carried out by fixing the two ends of the sensing head on two translation stages and moving inward one stage to change the separation between them, so that different bend radii could be applied on the fiber sensor. The sensor's bend curvature is given by $c=2 h /\left(h^{2}+L^{2}\right)$, where $h$ is the bend displacement at the center of the sensor, $c$ is the bend curvature, and $L$ is the half of the distance between the two stages.

The bend sensitivity is dependent on the bend direction because the SHF-LPG was side-inscripted, and the fiber cross-section is not symmetrical. During $\mathrm{CO}_{2}$ laser inscription, the index change on the incident side of the fiber is larger than that on the escape side because the light of both the incident side and escape side is different [17]. Asymmetric index change within the cross-section of the fiber leads to the bend sensitivity of the $\mathrm{CO}_{2}$ laser induced LPG depending on its inscription orientation strongly. Before measurement, the incident side of the SHF-LPG was put upward straightly and set as its initial position marked as $0^{\circ}$. Different bend directions were measured by clockwise rotation SHF.

Figure 6 shows measured transmission spectra of the SHF-LPG under different directions and bend curvatures. It can be seen obviously that the resonant wavelength and amplitude are different for different orientations of $0^{\circ}$ and $180^{\circ}$ even under the same curvature of $0.75 \mathrm{~m}^{-1}$. So the bend sensitivity of this SHF-LPG depends on its bend orientation strongly as aforementioned. Experimental results show that the incident direction is most sensitive to bend by adjusting the angle gradually. Figure 7 (a) shows several transmission spectra of the sensor head with different bend curvatures at the incident direction. The resonant wavelength red shifts from $1534.7 \mathrm{~nm}$ to $1541.2 \mathrm{~nm}$, and the amplitude decreases from $-17.21 \mathrm{~dB}$ to $-7.57 \mathrm{~dB}$ with bend increasing from $0.644 \mathrm{~m}^{-1}$ to $1.288 \mathrm{~m}^{-1}$. It can be explained that bend of the SHF-LPG leads to waveguide structure changes and axial strain. Waveguide structure changes result in the mode field distribution variation of fiber guided mode. Axial strain

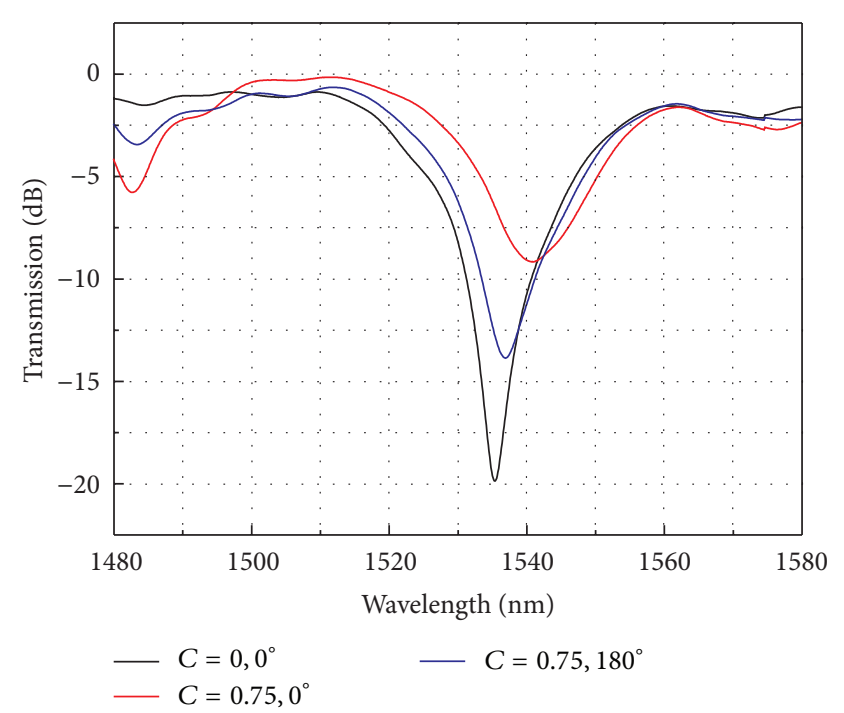

FIgURE 6: Transmission spectra of the SHF-LPG under different directions.

results in the effective RI variation of fiber. Figure 7(b) shows the relationship between bend curvature and the resonant wavelength. The achieved bend sensitivity is $\sim 9.36 \mathrm{~nm} /{ }^{\circ} \mathrm{C}$ with $R$-squared value of 0.98 . The sensitivity is relatively higher than that, $\sim 7 \mathrm{~nm} / \mathrm{m}^{-1}$, of the SMF-LPG written by using $\mathrm{CO}_{2}$ laser.

\section{Conclusion}

Long-period gratings (LPGs) have been fabricated in a sidehole fiber (SHF) by using a pulsed $\mathrm{CO}_{2}$ laser. Sensing characteristics of this SHF-LPG to temperature surrounding $\mathrm{RI}$ and bend have been investigated. Experimental results show that resonant wavelength of the SHF-LPG has a blue shift with temperature with sensitivity of $-0.11 \mathrm{~nm} /{ }^{\circ} \mathrm{C}$, which is about 2 times higher than that of SMF-LPG written by using $\mathrm{CO}_{2}$ laser and 1.35 times higher than that of mechanicallyintroduced SHF-LPG. It also has a blue shift with increasing sensitivity with surrounding RI ranging from 1.335 to 1.44 and a red shift with bending-direction-dependent sensitivity up to $9.36 \mathrm{~nm} / \mathrm{m}^{-1}$, which is relatively higher than that of normal 


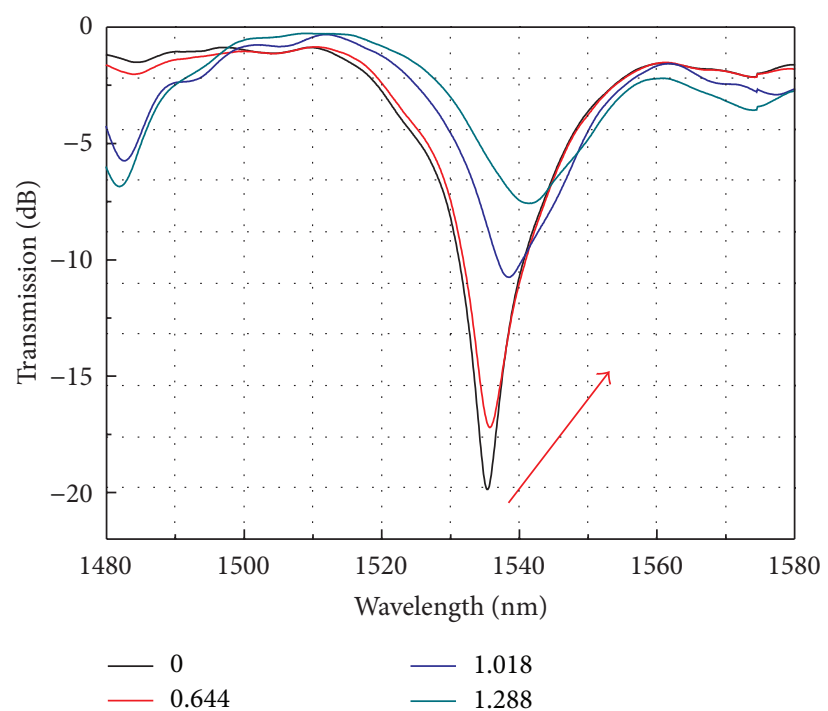

(a)

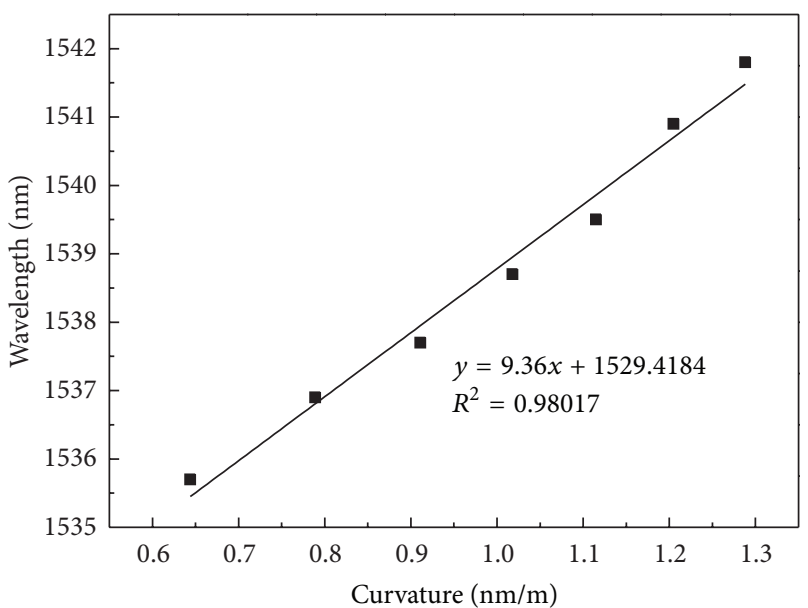

(b)

FIgURE 7: (a) Transmission spectra of the SHF-LPG under different curvatures at the incident direction. (b) Resonant wavelength versus curvature.

SMF-LPGs. These superior sensing characteristics provide a basis for its application in optic fiber sensing areas.

\section{Acknowledgments}

This work was supported by the National Basic Research Program of China (973 Program) under Grant no. 2010CB327804, National Natural Science Foundation of Zhejiang Province, China, under Grant no. Z13F050003, National Natural Science Foundation of China under Grants nos. 61007050 and 61007051, and Zhejiang Xinmiao Talent Project Grant no. 2012R409056. The authors acknowledge ACREO, Sweden, for providing them with the sample of side-hole fiber.

\section{References}

[1] H. M. Xie, P. Dabkiewicz, and R. Ulrich, "Side-hole fiber for fiber-optic pressure sensing," Optics Letters, vol. 11, no. 5, pp. 333-335, 1986.

[2] J. R. Clowes, S. Syngellakis, and M. N. Zervas, "Pressure sensitivity of side-hole optical fiber sensors," IEEE Photonics Technology Letters, vol. 10, no. 6, pp. 857-859, 1998.

[3] D. S. Moon, B. H. Kim, A. Lin et al., "The temperature sensitivity of Sagnac loop interferometer based on polarization maintaining side-hole fiber," Optics Express, vol. 15, no. 13, pp. 7962-7967, 2007.

[4] S. H. Lee, B. H. Kim, and W.-T. Han, "Effect of filler metals on the temperature sensitivity of side-hole fiber," Optics Express, vol. 17, no. 12, pp. 9712-9717, 2009.

[5] G. Chesini, C. M. B. Cordeiro, C. J. S. De Matos, M. Fokine, I. C. S. Carvalho, and J. C. Knight, "All-fiber devices based on photonic crystal fibers with integrated electrodes," Optics Express, vol. 17, no. 3, pp. 1660-1665, 2009.

[6] J. Tian, Q. Zhang, T. Fink et al., "Tuning operating point of extrinsic Fabry-Perot interferometric fiber-optic sensors using microstructured fiber and gas pressure," Optics Letters, vol. 37, no. 22, pp. 4672-4674, 2012.

[7] Y. Xin, X. Y. Dong, Q. Q. Meng et al., "Alcohol-filled side-hole fiber Sagnac interferometer for temperature measurement," Sensors and Actuators A, vol. 193, pp. 182-185, 2013.

[8] B. H. Kim, S. Moon, U.-C. Paek, and W.-T. Han, "All fiber polarimetric modulation using an electro-optic fiber with internal $\mathrm{Pb}$ Sn electrodes," Optics Express, vol. 14, no. 23, pp. 11234-11241, 2006.

[9] W. Zhou, Y. Zhou, X. Dong et al., "Fiber-optic curvature sensor based on cladding-mode bragg grating excited by fiber multimode interferometer," Photonic Journal, vol. 4, no. 3, pp. 10511057, 2012.

[10] H. Bao, X. Dong, L.-Y. Shao, C.-L. Zhao, C. C. Chan, and P. Shum, "Temperature-insensitive 2-D pendulum clinometer using two fiber bragg gratings," IEEE Photonics Technology Letters, vol. 22, no. 12, pp. 863-865, 2010.

[11] X. Dong, Y. Jin, W. Zhou, J. Zhou, and P. Shum, "Simultaneous measurement of strain and temperature with side-hole fiber based Bragg grating," in Proceedings of the 7th International Conference on Information, Communications and Signal Processing (ICICS '09), December 2009.

[12] E. Chmielewska, W. Urbańczyk, and W. J. Bock, "Measurement of pressure and temperature sensitivities of a Bragg grating imprinted in a highly birefringent side-hole fiber," Applied Optics, vol. 42, no. 31, pp. 6284-6291, 2003.

[13] Y. X. Jin, C. C. Chan, Y. F. Zhang, and X. Y. Dong, "Mechanically induced long-period fiber grating in side-hole single-mode fiber for temperature and refractive sensing," Optics Communications, vol. 283, no. 7, pp. 1303-1306, 2010.

[14] J. M. P. Coelho, M. C. Nespereira, M. Abreu, and J. M. Rebordao, "Modeling refractive index change in writing long-period fiber gratings using mid-infrared laser radiation," Photonic Sensor, vol. 3, no. 1, pp. 67-73.

[15] Y. Zhangwei, W. Margulis, O. Tarasenko, H. Knape, and P.Y. Fonjallaz, "Nanosecond switching of fiber Bragg gratings," Optics Express, vol. 15, no. 22, pp. 14948-14953, 2007. 
[16] T. W. MacDougall, S. Pilevar, C. W. Haggans, and M. A. Jackson, "Generalized expression for the growth of long period gratings," IEEE Photonics Technology Letters, vol. 10, no. 10, pp. 1449-1451, 1998.

[17] Y.-J. Rao, Y.-P. Wang, Z.-L. Ran, and T. Zhu, "Novel fiber-optic sensors based on long-period fiber gratings written by highfrequency $\mathrm{CO}_{2}$ laser pulses," Journal of Lightwave Technology, vol. 21, no. 5, pp. 1320-1327, 2003.

[18] J. H. Chong, P. Shum, H. Haryono et al., "Measurements of refractive index sensitivity using long-period grating refractometer," Optics Communications, vol. 229, no. 1-6, pp. 65-69, 2004.

[19] H. J. Patrick, A. D. Kersey, and F. Bucholtz, "Analysis of the response of long period fiber gratings to external index of refraction," Journal of Lightwave Technology, vol. 16, no. 9, pp. 1606-1612, 1998. 

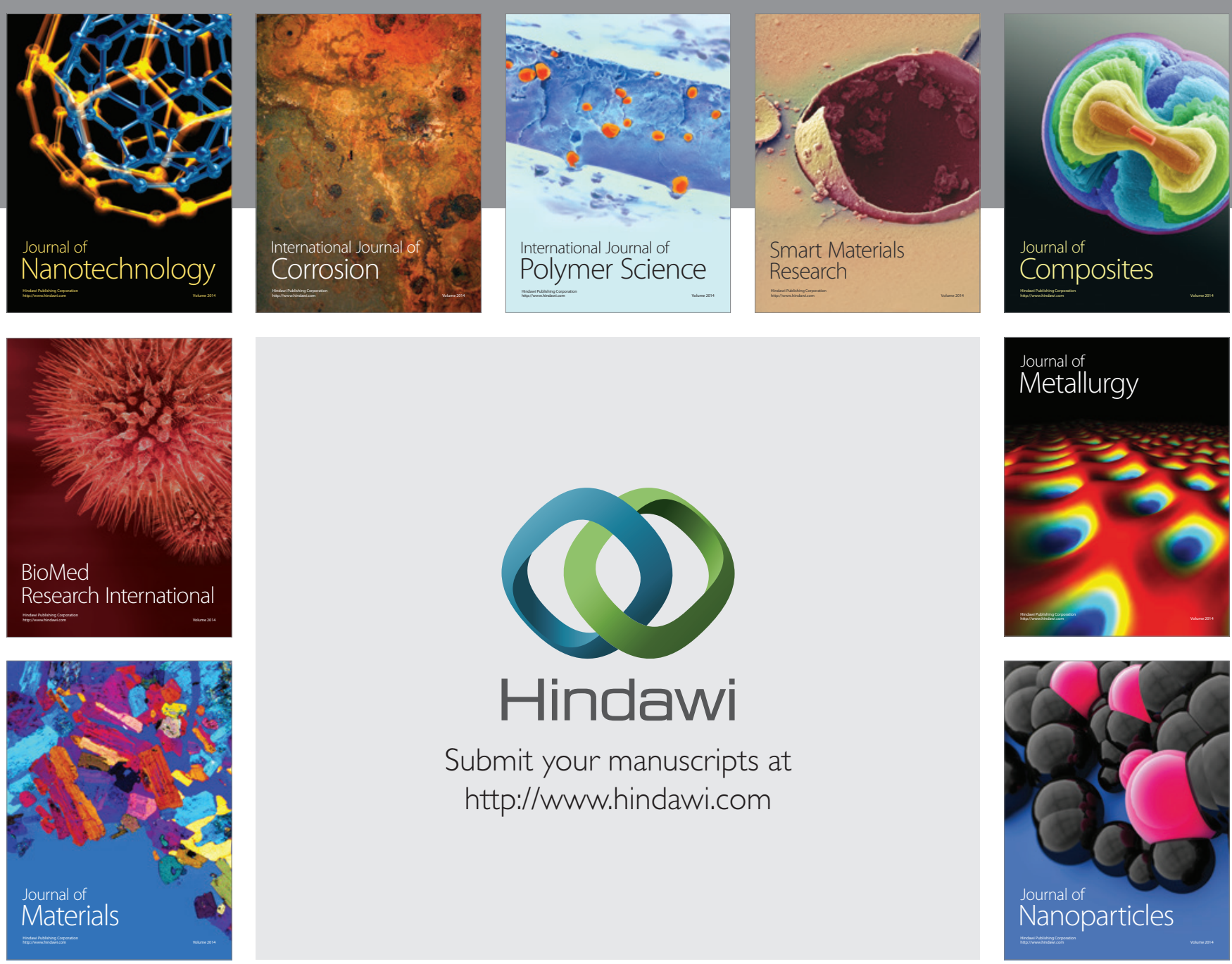

Submit your manuscripts at http://www.hindawi.com
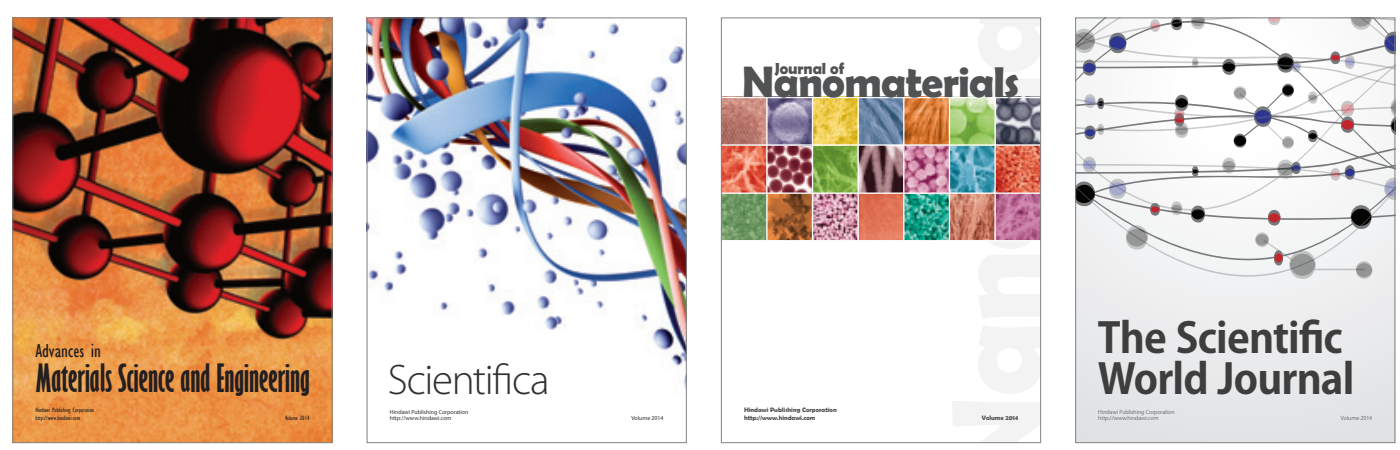

\section{The Scientific World Journal}
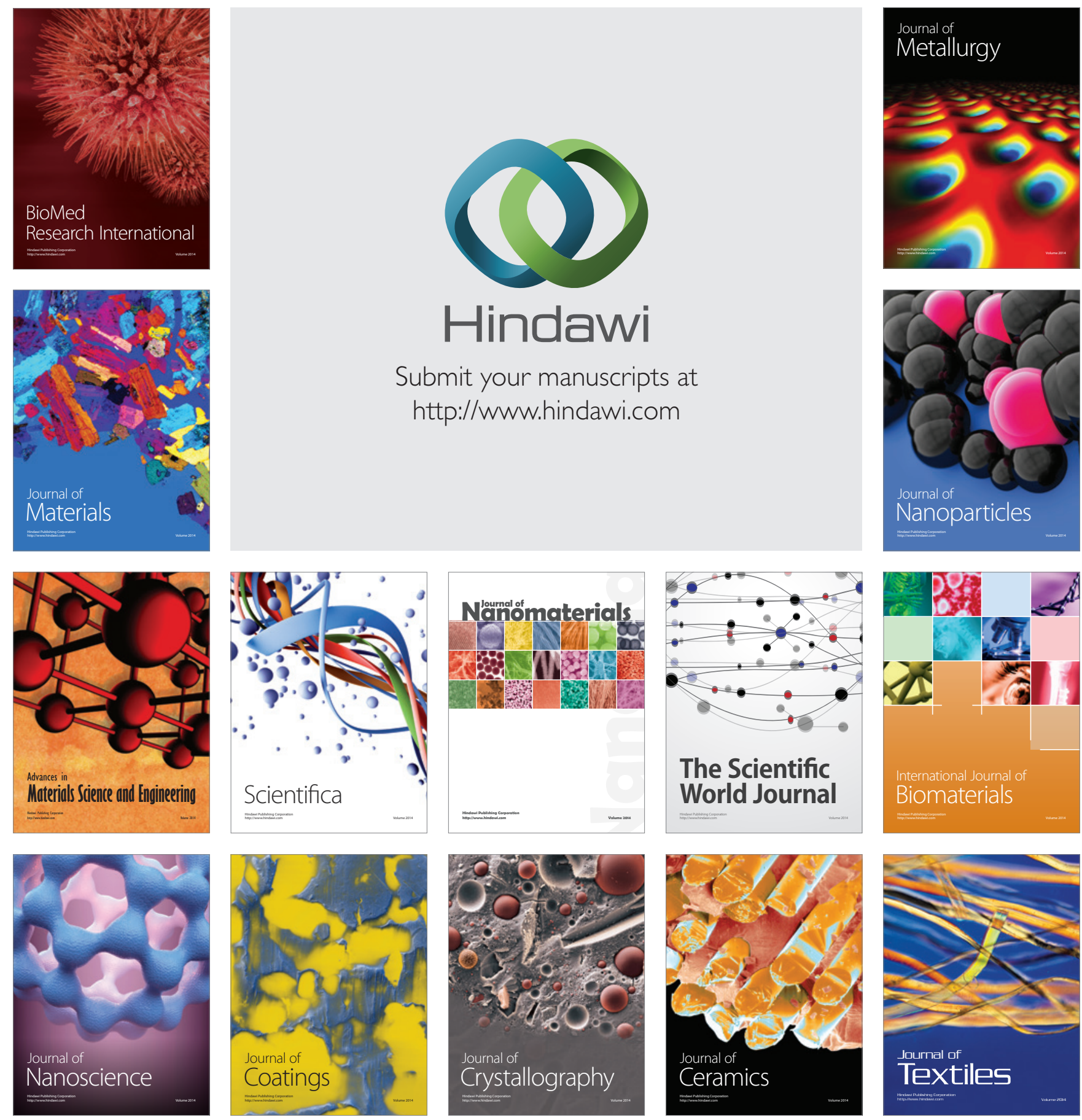